
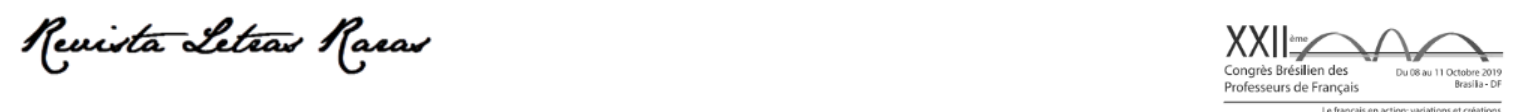

Actes du XXIlème Congrès brésilien des professeurs de français (dans Édition spéciale de la Revue Letras Raras 2020)

ISSN : 2317-2347 - v. 9, Dossier Spécial (2020)

Todo o conteúdo da RLR está licenciado sob Creative Commons Atribuição 4.0 Internacional

\title{
Des dynamiques coopératives au travail en réseau : une évolution nécessaire au service du développement professionnel ?
}

\section{Rana Challah*}

Docteure en sciences de l'éducation. Chercheure postdoctorale spécialisée en pédagogie de l'enseignement supérieur à l'Université Gustave Eiffel à Paris et enseignante au Département des sciences de l'éducation à l'université Rennes 2.

Reçu le 03 nov. 2020. Approuvé le 10 nov. 2020.

\section{Comment citer cet article:}

CHALLAH, Rana. Des dynamiques coopératives au travail en réseau: une évolution nécessaire au service du développement professionnel ?. In: CONGRES BRESILIEN DES PROFESSEURS DE FRANÇAIS, 22., 2019, Brasília. Actes du XXIIème Congrès Brésilien des Professeurs de Français. Édition spéciale de la Revue Letras Raras: Campina Grande. EDUFCG. nov. 2020, p. 474-487.

\section{RÉSUMÉ}

La coopération entre les enseignants, prônée par les textes officiels français (MEN, 2008, 2013) et par la recherche comme vecteur de développement professionnel est une thématique majeure dans les études sur la formation des enseignants (DIONNE, 2003 ; HARGREAVES ET FULLAN, 1998 ; HUBERMAN, 1995). Cette conférence plénière, qui a été proposée dans le cadre de l'axe Politiques publiques et mémoires partagées de la 22 ème édition du Congrès brésilien des professeurs de français à Brasilia en 2019 a trois objectifs. D'abord, présenter quelques éléments théoriques reflétant l'importance de la coopération et son impact sur le développement professionnel des enseignants. Ensuite, présenter les résultats d'une enquête par questionnaire $(\mathrm{N}=423)$ menée au sein d'un des réseaux de l'AEFE. Celle-ci reflète les représentations des enseignants quant aux processus, aux contenus et à l'impact du travail en équipe sur leurs pratiques. Et finalement, proposer quelques pistes qui assurent les degrés de coopération et l'importance de l'instauration d'une culture d'interdépendance au sein des établissements scolaires.

MOTS-CLÉS : Coopération ; Travail en réseau ; Développement professionnel ; Enseignants.

\section{Introduction}

Dans un contexte de changements et de réformes, l'institution scolaire met en place des dispositifs pour aider l'enseignant à développer ses capacités d'analyse, qui lui permettent

$*$

$\triangle$ ranachallah@gmail.com 

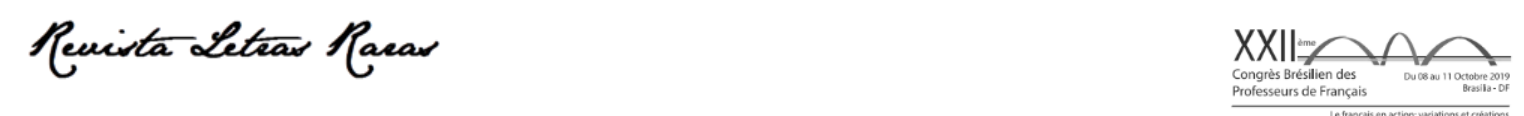

Actes du XXIlème Congrès brésilien des professeurs de français (dans Édition spéciale de la Revue Letras Raras 2020)

ISSN : 2317-2347 - v. 9, Dossier Spécial (2020)

Todo o conteúdo da RLR está licenciado sob Creative Commons Atribuição 4.0 Internacional

d'interpréter les informations qu'il reçoit et de pouvoir les traduire en des stratégies d'enseignement adaptées au contexte et aux élèves. Il est enjoint de produire de nouvelles connaissances et d'ajuster ses pratiques pour parvenir à aider les élèves à apprendre à apprendre. Ainsi, il est censé être un créateur de sens et de savoir. De nombreux travaux (LAFORTUNE ; LEPAGE, 2008 ; LECLERC, 2012 ; FULLAN ; HARGREAVES, 2012) montrent que la complexité du travail à produire par l'enseignant, et les capacités qu'il doit développer ne peuvent plus se faire de manière isolée. Si les dynamiques coopératives peuvent favoriser le développement professionnel de l'enseignant, quelles formes de coopération sont les plus susceptibles de garantir un développement professionnel possible et continu de l'enseignant ? En effet, les dynamiques coopératives se caractérisent par plusieurs niveaux : la coordination, la coopération, la collaboration, les communautés d'apprentissage professionnelles et le travail en réseau. Quels types et quels niveaux de coopération favorisent le développement professionnel des enseignants et l'efficacité des établissements ? Ces formes de travail collectif contribuent-elles à l'émergence d'une " culture d'interactivité » ? Peut-on passer d'un paradigme de " coopération juxtapositive » à un paradigme de "réseautage " ? L'objectif de cette conférence est d'analyser les spécificités de ces dynamiques et les contraintes qui peuvent empêcher ou, au contraire, favoriser le développement professionnel de l'enseignant.

\section{Les dynamiques coopératives sont-elles indispensables ? État de la littérature scientifique}

Pourquoi cet engouement autour des dynamiques coopératives ? En effet, l'étude du travail enseignant montre que ce dernier est enjoint de coopérer avec plusieurs acteurs au sein de l'établissement scolaire (MEN, 2008, 2013), cette coopération affectant son activité individuelle (MARCEL et al., 2007 ; TARDIF ; LESSARD, 1999).

Les travaux de Prieur, Sanchez et Aldon (2011) montrent que les dynamiques coopératives entre les enseignants permettent aux élèves de mieux percevoir la complémentarité qui existe entre les disciplines et d'appréhender la nécessité d'un enseignement décloisonné. De même, les travaux de Grangeat (2008) montrent que le travail collectif enseignant contribue à l'amélioration de la professionnalité enseignante grâce à la dynamique de recherche et d'auto-formation engagée. Ces auteurs pensent que le travail collectif améliore également la relation administration-enseignant, 

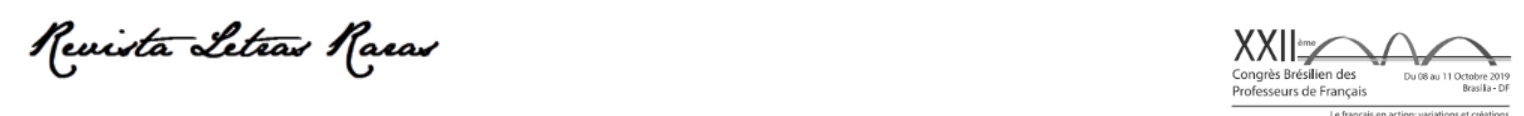

Actes du XXIlème Congrès brésilien des professeurs de français (dans Édition spéciale de la Revue Letras Raras 2020) ISSN : 2317-2347 - v. 9, Dossier Spécial (2020)

Todo o conteúdo da RLR está licenciado sob Creative Commons Atribuição 4.0 Internacional

favorise l'émergence de la culture de partage entre les enseignants et permet de mieux aider les élèves dans leur choix d'orientation professionnelle.

Quant à l'impact des dynamiques coopératives sur le développement professionnel, de nombreux chercheurs (DAY, 1999 ; HARGREAVES et FULLAN, 1998 ; DIONNE, 2003) considèrent que le développement professionnel est un processus dynamique qui se nourrit de l'interaction avec les pairs. Selon Hargreaves et Fullan (1998), la manière dont les enseignants travaillent ensemble influence la qualité de leur travail avec les élèves. En effet, il semble que l'instauration d'une culture de coopération renforce le sentiment d'avoir un but commun et le degré d'adaptabilité aux changements. À cet égard, il existe différents degrés de coopération qui présentent de nombreux avantages. Mais avant de les exposer, nous allons revenir sur le niveau de coopération qui peut le mieux favoriser le développement professionnel des enseignants travaillant au sein d'un réseau de l'AEFE.

\section{Méthodologie}

Compte tenu de l'objectif de la conférence, nous présentons une partie du volet quantitatif de la recherche. Cette partie concerne essentiellement les rubriques qui permettent de mieux comprendre les modalités de l'organisation du travail collectif des enseignants et les représentations des enseignants quant à l'impact de ce travail sur leurs pratiques.

\subsection{Méthode d'analyse statistique adoptée}

Afin d'analyser le questionnaire, des méthodes de statistique descriptive et inférentielle ont été adoptées. Dans un premier temps, les données du questionnaire contenant les modalités représentant les avis des enseignants ont été regroupées, puis codées selon l'échelle Likert et ensuite calculées avec l'appui d'un logiciel statistique SPSS, spécialisé en traitement de données quantitatives et statistiques. Une fois le codage fait, nous avons calculé des moyennes et l'écart-type par établissement, genre, champ disciplinaire, fonction, années d'expérience dans l'enseignement et 

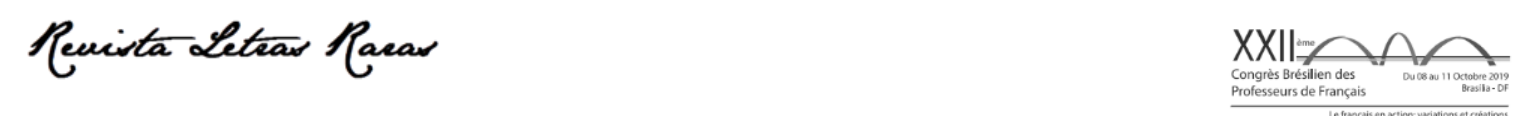

Actes du XXIlème Congrès brésilien des professeurs de français (dans Édition spéciale de la Revue Letras Raras 2020)

ISSN : 2317-2347 - v. 9, Dossier Spécial (2020)

Todo o conteúdo da RLR está licenciado sob Creative Commons Atribuição 4.0 Internacional

années d'expérience dans l'établissement ${ }^{1}$. Compte tenu de la visée de la recherche, le test utilisé pour estimer la présence ou l'absence de différences significatives selon les variables retenues est celui de l'analyse de variance. L'analyse de variance MANOVA, à mesures répétées, permet d'analyser les différences entre les établissements, selon les critères susmentionnés. Ainsi, les données issues du questionnaire ont été analysées grâce à des procédés statistiques descriptifs et probabilistes. Les moyennes obtenues ont finalement permis une analyse comparative des différences entre les établissements enquêtés.

4 Résultats : les représentations des enseignants quant aux processus, aux contenus et à l'impact du travail en équipe sur leurs pratiques

Le Tableau 1 présente les cotes moyennes concernant la perception des enseignants du travail en équipe en ordre descendant, selon la cote globale obtenue par l'établissement, ainsi que les écarts-types et les effectifs et ce, pour chaque variable concernant le travail en équipe : processus, contenu et impact. L'analyse multivariée de ces données démontre qu'il existe une différence significative entre les établissements quant à cette variable $(F=4.038 ; d l=27 ; p<.001)$.

Une analyse des cotes moyennes relatives aux trois variables du travail en équipe prises séparément montre que les différences entre établissements existent pour les variables de processus $(F=7.574 ; d l=9 ; p<.001)$ et de contenu du travail en équipe $(F=5.362 ; d l=9 ; p<.001)$. En revanche, des différences sont perceptibles quant à l'impact du travail en équipe, mais n'atteignent le niveau habituel de signification ( $F=1.895 ; d l=9$; n.s.).

Tableau 1: Cotes moyennes, écarts-types et effectifs concernant la perception des enseignants du " travail en équipe : processus, contenus, impact », relatifs à la variable concernant le travail en équipe, selon l'établissement en ordre descendant de la cote globale :

1 Compte tenu de l'objectif de cette communication, nous avons fait le choix de présenter les différences entre les établissements uniquement. 
Actes du XXIlème Congrès brésilien des professeurs de français (dans Édition spéciale de la Revue Letras Raras 2020) ISSN : 2317-2347 - v. 9, Dossier Spécial (2020)

Todo o conteúdo da RLR está licenciado sob Creative Commons Atribuição 4.0 Internacional

\begin{tabular}{|c|c|c|c|c|c|c|c|c|c|c|c|c|}
\hline \multirow[b]{2}{*}{ Établissement } & \multicolumn{3}{|c|}{$\begin{array}{l}\text { Travail en équipe : } \\
\text { processus }\end{array}$} & \multicolumn{3}{|c|}{$\begin{array}{l}\text { Travail en équipe : } \\
\text { production et contenu }\end{array}$} & \multicolumn{3}{|c|}{$\begin{array}{c}\text { Impact du travail en } \\
\text { équipe }\end{array}$} & \multicolumn{3}{|c|}{ Cote globale } \\
\hline & $\mathbf{M} \& \mathbf{S}^{2}$ & SHS $^{3}$ & $A \& S^{4}$ & M\&S & SHS & A\&S & M\&S & SHS & A\&S & M\&S & SHS & A\&S \\
\hline$A$ & $\begin{array}{c}72.2 \\
(26.9) \\
\mathrm{N}=5\end{array}$ & $\begin{array}{c}83.3 \\
(16.1) \\
\mathrm{N}=6\end{array}$ & $\begin{array}{c}86.1 \\
(11.8) \\
\mathrm{N}=2\end{array}$ & $\begin{array}{c}62.5 \\
(37.5) \\
\mathrm{N}=5\end{array}$ & $\begin{array}{c}66.7 \\
(21.9) \\
\mathrm{N}=6\end{array}$ & $\begin{array}{c}93.8 \\
(8.83) \\
\mathrm{N}=2\end{array}$ & $\begin{array}{c}66 \\
(20.7) \\
\mathrm{N}=5\end{array}$ & $\begin{array}{c}95 \\
(8.4) \\
\mathrm{N}=6\end{array}$ & $\begin{array}{c}100 \\
(0) \\
\mathrm{N}=2\end{array}$ & 66.9 & 81.7 & 93.3 \\
\hline B & $\begin{array}{c}82.1 \\
(12.6) \\
\mathrm{N}=22\end{array}$ & $\begin{array}{c}87.4 \\
(13.4) \\
N=23\end{array}$ & $\begin{array}{c}85.2 \\
(12.4) \\
\mathrm{N}=12\end{array}$ & $\begin{array}{c}71.6 \\
(24.5) \\
\mathrm{N}=22\end{array}$ & $\begin{array}{c}68.5 \\
(22.6) \\
\mathrm{N}=23\end{array}$ & $\begin{array}{c}62.5 \\
(27.7) \\
\mathrm{N}=12\end{array}$ & $\begin{array}{c}86.8 \\
(19.9) \\
\mathrm{N}=22\end{array}$ & $\begin{array}{c}80.4 \\
(30) \\
\mathrm{N}=23\end{array}$ & $\begin{array}{c}87.5 \\
(12.1) \\
\mathrm{N}=12\end{array}$ & 80.2 & 78.8 & 78.4 \\
\hline C & $\begin{array}{c}81 \\
(17.8) \\
\mathrm{N}=14\end{array}$ & $\begin{array}{c}84.3 \\
(12.5) \\
\mathrm{N}=18\end{array}$ & $\begin{array}{l}80.6 \\
(3.9) \\
\mathrm{N}=2\end{array}$ & $\begin{array}{c}67.9 \\
(19.4) \\
N=14\end{array}$ & $\begin{array}{c}80.6 \\
(19.8) \\
N=18\end{array}$ & $\begin{array}{c}62.5 \\
(17.7) \\
\mathrm{N}=2\end{array}$ & $\begin{array}{c}71.4 \\
(32.3) \\
\mathrm{N}=14\end{array}$ & $\begin{array}{c}76.1 \\
(22.5) \\
\mathrm{N}=18\end{array}$ & $\begin{array}{c}70 \\
(28.3) \\
\mathrm{N}=2\end{array}$ & 73.4 & 80.3 & 71 \\
\hline $\mathrm{D}$ & $\begin{array}{c}62.8 \\
(22) \\
\mathrm{N}=20\end{array}$ & $\begin{array}{c}66.7 \\
(19.9) \\
\mathrm{N}=41\end{array}$ & $\begin{array}{c}83.3 \\
(13.6) \\
\mathrm{N}=4\end{array}$ & $\begin{array}{c}55.6 \\
(27) \\
\mathrm{N}=20\end{array}$ & $\begin{array}{c}56.1 \\
(22.9) \\
\mathrm{N}=41\end{array}$ & $\begin{array}{c}81.3 \\
(29.8) \\
\mathrm{N}=4\end{array}$ & $\begin{array}{c}71 \\
(25.1) \\
\mathrm{N}=20\end{array}$ & $\begin{array}{c}73.7 \\
(27.9) \\
\mathrm{N}=41\end{array}$ & $\begin{array}{l}92.5 \\
(15) \\
\mathrm{N}=4\end{array}$ & 63.1 & 65.5 & 85.7 \\
\hline$E$ & $\begin{array}{c}72.2 \\
(13.3) \\
\mathrm{N}=17\end{array}$ & $\begin{array}{c}74.3 \\
(15.7) \\
\mathrm{N}=24\end{array}$ & $\begin{array}{c}69.4 \\
(27.5) \\
\mathrm{N}=2\end{array}$ & $\begin{array}{c}75 \\
(17.1) \\
N=17\end{array}$ & $\begin{array}{c}59.4 \\
(22.8) \\
\mathrm{N}=24\end{array}$ & $\begin{array}{c}50 \\
(0) \\
\mathrm{N}=2\end{array}$ & $\begin{array}{c}80.6 \\
(14.3) \\
\mathrm{N}=17\end{array}$ & $\begin{array}{c}79.6 \\
(19.4) \\
N=24\end{array}$ & $\begin{array}{c}70 \\
(14.1) \\
\mathrm{N}=2\end{array}$ & 75.9 & 71.1 & 63.1 \\
\hline$F$ & $\begin{array}{c}67.3 \\
(17.2) \\
\mathrm{N}=18\end{array}$ & $\begin{array}{c}68.6 \\
(19) \\
\mathrm{N}=37\end{array}$ & $\begin{array}{l}70.8 \\
(5.3) \\
\mathrm{N}=4\end{array}$ & $\begin{array}{c}59 \\
(18.1) \\
N=18\end{array}$ & $\begin{array}{c}46.6 \\
(20.5) \\
\mathrm{N}=37\end{array}$ & $\begin{array}{l}59.4 \\
(6.3) \\
\mathrm{N}=4\end{array}$ & $\begin{array}{c}78.9 \\
(30.5) \\
\mathrm{N}=18\end{array}$ & $\begin{array}{c}76.5 \\
(22.8) \\
\mathrm{N}=37\end{array}$ & $\begin{array}{c}42.5 \\
(26.3) \\
\mathrm{N}=4\end{array}$ & 68.4 & 63.9 & 57.6 \\
\hline G & $\begin{array}{c}63.9 \\
(19.8) \\
\mathrm{N}=14\end{array}$ & $\begin{array}{c}68.3 \\
(18.6) \\
\mathrm{N}=17\end{array}$ & $\begin{array}{c}44.4 \\
(-) \\
\mathrm{N}=1\end{array}$ & $\begin{array}{c}54.5 \\
(18.1) \\
\mathrm{N}=14\end{array}$ & $\begin{array}{c}46.3 \\
(24.5) \\
\mathrm{N}=17\end{array}$ & $\begin{array}{c}62.5 \\
(-) \\
\mathrm{N}=1\end{array}$ & $\begin{array}{c}90 \\
(17.5) \\
\mathrm{N}=14\end{array}$ & $\begin{array}{c}82.9 \\
(22.3) \\
\mathrm{N}=17\end{array}$ & $\begin{array}{c}100 \\
(-) \\
N=1\end{array}$ & 69.5 & 65.8 & 69 \\
\hline $\mathrm{H}$ & $\begin{array}{c}57.4 \\
(25.7) \\
\mathrm{N}=12\end{array}$ & $\begin{array}{c}67.4 \\
(18.9) \\
N=16\end{array}$ & $\begin{array}{c}55.6 \\
(23.6) \\
\mathrm{N}=2\end{array}$ & $\begin{array}{c}43.8 \\
(26.9) \\
\mathrm{N}=12\end{array}$ & $\begin{array}{c}57 \\
(29.2) \\
N=16\end{array}$ & $\begin{array}{c}43.8 \\
(61.9) \\
\mathrm{N}=2\end{array}$ & $\begin{array}{c}65 \\
(32.6) \\
N=12\end{array}$ & $\begin{array}{c}88.1 \\
(16.8) \\
\mathrm{N}=16\end{array}$ & $\begin{array}{c}70 \\
(28.3) \\
\mathrm{N}=2\end{array}$ & 55.4 & 70.8 & 56.5 \\
\hline I & $\begin{array}{c}70.8 \\
(20.7) \\
\mathrm{N}=8\end{array}$ & $\begin{array}{l}80.2 \\
(7.4) \\
\mathrm{N}=9\end{array}$ & $\begin{array}{c}55.6 \\
(23.6) \\
\mathrm{N}=2\end{array}$ & $\begin{array}{c}53.1 \\
(25.7) \\
\mathrm{N}=8\end{array}$ & $\begin{array}{c}55.6 \\
(12.7) \\
\mathrm{N}=9\end{array}$ & $\begin{array}{c}31.2 \\
(26.5) \\
\mathrm{N}=2\end{array}$ & $\begin{array}{c}81.3 \\
(18.1) \\
\mathrm{N}=8\end{array}$ & $\begin{array}{c}80 \\
(20.6) \\
\mathrm{N}=9\end{array}$ & $\begin{array}{c}55 \\
(7.1) \\
\mathrm{N}=2\end{array}$ & 68.4 & 71.9 & 47.3 \\
\hline$J$ & $\begin{array}{c}63.9 \\
(18) \\
\mathrm{N}=16\end{array}$ & $\begin{array}{c}71.3 \\
(18.2) \\
\mathrm{N}=30\end{array}$ & $\begin{array}{l}79.6 \\
(16) \\
N=3\end{array}$ & $\begin{array}{c}57 \\
(19.9) \\
N=16\end{array}$ & $\begin{array}{c}56.3 \\
(20.2) \\
\mathrm{N}=30\end{array}$ & $\begin{array}{c}54.2 \\
(31.5) \\
\mathrm{N}=3\end{array}$ & $\begin{array}{c}82.5 \\
(15.7) \\
N=16\end{array}$ & $\begin{array}{c}85 \\
(20.8) \\
N=30\end{array}$ & $\begin{array}{c}93.3 \\
(11.5) \\
\mathrm{N}=3\end{array}$ & 67.8 & 70.9 & 75.7 \\
\hline
\end{tabular}

2 Math et sciences

3 Sciences humaines et sociales

${ }^{4}$ Arts et sports 

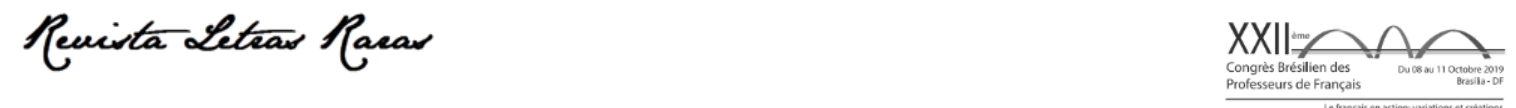

Actes du XXIlème Congrès brésilien des professeurs de français (dans Édition spéciale de la Revue Letras Raras 2020)

ISSN : 2317-2347 - v. 9, Dossier Spécial (2020)

Todo o conteúdo da RLR está licenciado sob Creative Commons Atribuição 4.0 Internacional

\begin{tabular}{|c|c|c|c|c|c|c|c|c|c|c|c|c|}
\hline & 69.3 & 73.2 & 77 & 61 & 57.7 & 61.8 & 78.5 & 79.8 & 79.4 & & \\
TOTAL & $\begin{array}{c}(19.8) \\
\mathrm{N}=146\end{array}$ & $\begin{array}{c}(18.5) \\
\mathrm{N}=221\end{array}$ & $\begin{array}{c}(16.7) \\
\mathrm{N}=34\end{array}$ & $\begin{array}{c}(23.8) \\
\mathrm{N}=146\end{array}$ & $\begin{array}{c}(23.7) \\
\mathrm{N}=221\end{array}$ & $\begin{array}{c}(27.3) \\
\mathrm{N}=34\end{array}$ & $\begin{array}{c}(24.2) \\
\mathrm{N}=146\end{array}$ & $\begin{array}{c}(22.9) \\
\mathrm{N}=221\end{array}$ & $\begin{array}{c}(22.7) \\
\mathrm{N}=34\end{array}$ & & \\
\end{tabular}

Source : Tableau organisé par l'auteure (2016)

L'examen de la moyenne générale des cotes globales obtenues dénote une appréciation élevée de l'impact du travail en équipe (79.3) avec peu de variation d'un établissement à l'autre (minimum : 73.8\% ; maximum: 86.6\%), suivie par une appréciation moins élevée concernant le processus du travail en équipe (72.2), alors que la moyenne des cotes globales concernant les contenus du travail en équipe reflète une appréciation paradoxalement plus basse (59.3) et à un niveau inférieur au pourcentage reflétant une appréciation positive de $66.6 \%$, soit une cote de 2 sur 3 , pour sept des dix établissements, dénotant une réelle insatisfaction quant aux contenus abordés dans le travail en équipe.

Par ailleurs, l'examen des cotes moyennes globales accordées par les sujets aux trois variables du travail en équipe (voir tab. 1) permet de classer l'appréciation des enseignants selon trois niveaux. Une appréciation élevée est notée dans trois établissements dont la cote globale s'établit respectivement à $79.2,77.8$ et $76.9 \%$ celle-ci est suivie par une appréciation relativement élevée qui concerne les deux établissements dont la cote globale se situe à 72.5 et 70.2 respectivement ; alors que l'appréciation des enseignants du travail en équipe pour les cinq derniers établissements se situe à un niveau relativement faible (cotes moyennes globales variant entre 67.9 et 63.7).

L'examen des cotes moyennes accordées par les sujets à la variable relative au processus du travail en équipe permet de classer l'appréciation des enseignants en trois catégories. Une appréciation très élevée est notée pour deux établissements dont la cote globale est respectivement de 84.9 et 82.7 ; celle-ci est suivie par une appréciation élevée qui concerne les trois établissements dont la cote globale se situe respectivement à 79.5 et 73 ; alors que l'appréciation des enseignants travaillant dans les cinq autres établissements se situe à un niveau relativement faible (cotes moyennes variant entre 69.4 et 62.6) et en dessous de la moyenne générale (72.2).

En outre, l'examen des cotes moyennes accordées par les sujets à la variable relative au contenu du travail en équipe permet de classer l'appréciation des enseignants en trois catégories. Une appréciation élevée est notée pour un établissement dont la cote globale s'établit à 74.3 ; celle-ci est 
Actes du XXIlème Congrès brésilien des professeurs de français (dans Édition spéciale de la Revue Letras Raras 2020) ISSN : 2317-2347 - v. 9, Dossier Spécial (2020)

Todo o conteúdo da RLR está licenciado sob Creative Commons Atribuição 4.0 Internacional

suivie par une appréciation minimalement positive qui concerne les trois établissements dont la cote globale est respectivement de 69.2 et 65.3; alors que l'appréciation des enseignants travaillant dans les six autres établissements se situe à un niveau plutôt négatif (cotes moyennes variant entre 57.5 et 50.4), la moyenne générale se situant à $59.3 \%$. Ces éléments sont illustrés dans le travail en équipe selon l'établissement en ordre descendant de la cote globale (Tab. 2).

Tableau 2: Résultats de l'analyse multivariée des cotes attribuées par les enseignants à la variable concernant le processus, contenu et impact du travail en équipe, selon l'établissement.

\begin{tabular}{|c|c|c|c|c|}
\hline Source de variance & Variable Dépendante & $\mathbf{F}$ & dl & Sig. \\
\hline \multirow{3}{*}{ Etablissement } & Processus & 7.574 & 9 & $p<.001(000)$ \\
\cline { 2 - 5 } & Contenu & 5.362 & 9 & $p<.001(.000)$ \\
\cline { 2 - 5 } & Impact & 1.895 & 9 & n.s. (.051) \\
\hline
\end{tabular}

Source : Tableau organisé par l'auteure (2016).

Figure 1 : Le travail en équipe selon l'établissement en ordre descendant de la cote globale

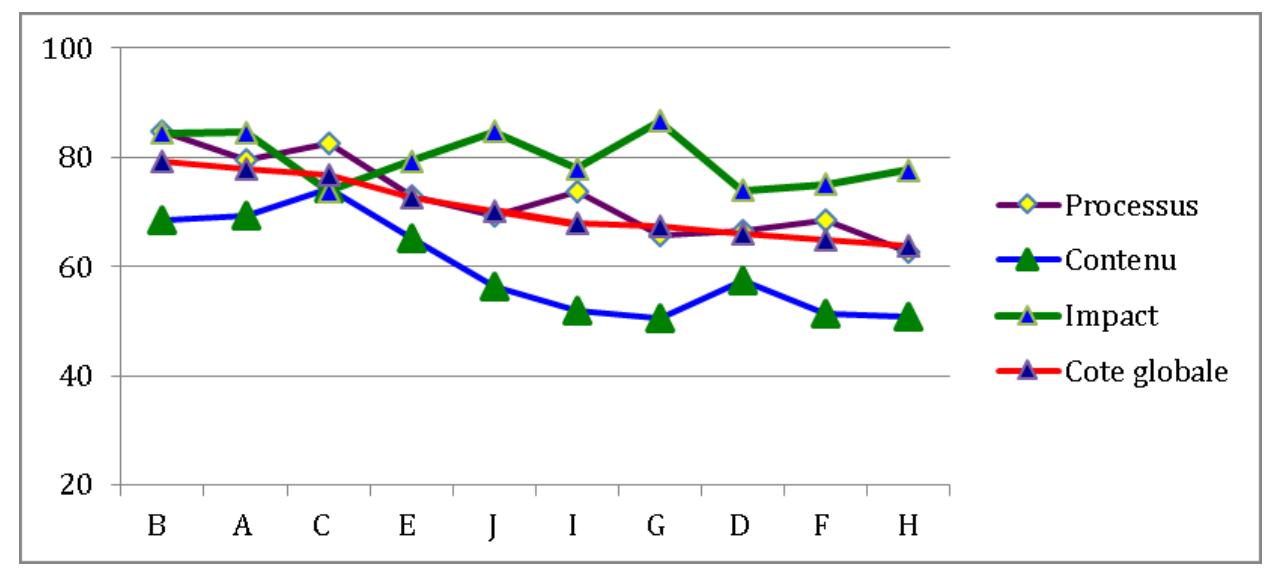



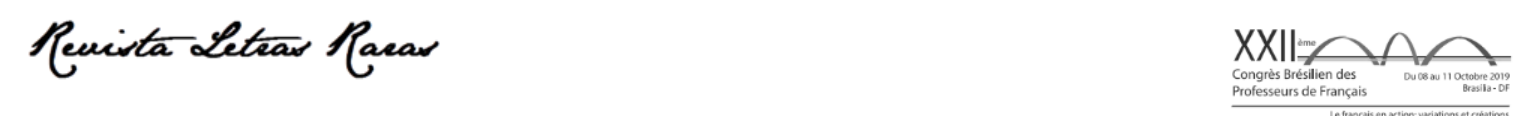

Actes du XXIlème Congrès brésilien des professeurs de français (dans Édition spéciale de la Revue Letras Raras 2020) ISSN : 2317-2347 - v. 9, Dossier Spécial (2020)

Todo o conteúdo da RLR está licenciado sob Creative Commons Atribuição 4.0 Internacional

Source : Figure organisée par l'auteure (2016).

\section{Le travail en équipe entre coordination disciplinaire et production collective : vers un niveau} de coopération mobilisateur et pérenne?

Pour ce qui concerne le travail en équipe, les résultats du questionnaire reflètent une appréciation minimalement positive des contenus du travail en équipe, une appréciation un peu plus élevée des processus inhérents à ce travail et, paradoxalement, une appréciation positive de l'impact de ce travail. Les données recueillies permettent de repérer trois formes :

- Le travail en équipe est le fruit d'une interaction entre des enseignants acteurs ayant un objectif commun, favorisant une production d'équipe. C'est le cas d'une minorité d'établissements, dans lesquels les enseignants considèrent que l'organisation ainsi que les processus du travail en équipe permettent à l'enseignant d'évoluer dans ses pratiques.

- Le travail en équipe se limite à des échanges plus ou moins formels dans le cadre des réunions disciplinaires, sans production collective. On peut se demander si ces échanges permettent vraiment aux enseignants d'évoluer dans leurs pratiques.

- Le travail en équipe se limite à la participation des enseignants aux réunions. Dans ce cas, qui concerne la majorité des établissements, les enseignants sont des acteurs passifs qui exécutent un travail prescrit et souvent individuel. Ce travail, souvent préparé par le coordonnateur de discipline peut, dans certains cas, contenir des nouvelles méthodes qui permettent aux enseignants d'avoir l'impression d'évoluer dans leurs pratiques.

Nous constatons que la première forme permet de repérer certains acquis et une progression professionnelle qui est le fruit d'une réflexion sur l'objet de travail alors que la deuxième forme semble contribuer à une évolution professionnelle individuelle mais relative. Quant à la troisième forme, elle semble moins aider réellement les enseignants à évoluer dans leurs pratiques. En effet, ces trois formes rappellent le modèle proposé par Bardram (1998) qui identifie trois degrés reflétant les dynamiques du travail coopératif : la coordination, la coopération et la construction collective de l'objet de l'activité. 

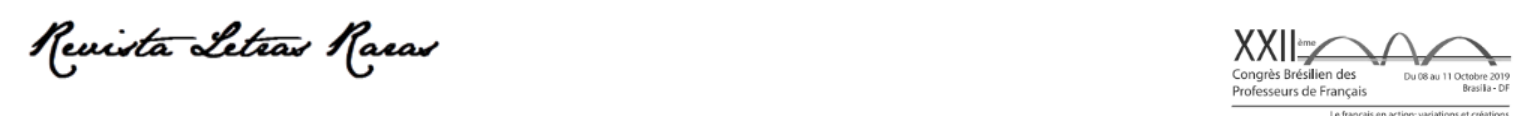

Actes du XXIlème Congrès brésilien des professeurs de français (dans Édition spéciale de la Revue Letras Raras 2020)

ISSN : 2317-2347 - v. 9, Dossier Spécial (2020)

Todo o conteúdo da RLR está licenciado sob Creative Commons Atribuição 4.0 Internacional

L'efficacité et la performance visées par les établissements scolaires supposent une " conjugaison dialectique de l'ordre et de la vie : la pyramide garantit l'ordre tandis que le réseau assure la vie » (SÉRIEYX et al., 1996, p. 13). À l'ère de la mondialisation, le travail d'un établissement scolaire doit s'inscrire dans un esprit d'interdépendance, dans la mesure où, il ne peut plus être dissocié de son environnement. En effet, " plus l'environnement devient évolutif, concurrentiel, imprévisible, plus se révèlent importantes les capacités de souplesse, de réactivité, de multiplication des intelligences en tant que facteurs d'innovations, et plus il devient nécessaire de "mettre du réseau dans sa pyramide" » (SÉRIEYX et al., 1996, p. 13) .

\section{Coordination, collaboration ou coopération : quels niveaux de coopération pour favoriser le changement?}

Les termes collaboration et coopération sont souvent utilisés de manière indistincte. C'est pourquoi il est nécessaire d'en préciser le sens. La coopération désigne la réunion des personnes qui ont pour objectif de réaliser une tâche avec une diligence et une réactivité mutuelles (Wildavsky, 1986). Pour Baudrit (2005), le travail coopératif est un travail dont l'objet est connu et partagé par les membres du groupe. II a une fonction instrumentale et peut être associé à l'apprentissage coopératif qui permet « d'optimiser les apprentissages de chacun » (BAUDRIT, 2005, p. 5) alors que le travail collaboratif suppose une compréhension mutuelle du point de vue de l'autre pour progresser. Little (1990, p. 520) considère que la collaboration est fondée sur des principes de synergie, " d'interdépendance » et de partage d'un but commun.

Il est important de préciser que, selon la littérature scientifique que nous allons présenter ciaprès, les dynamiques interactives ne peuvent pas se réduire à une simple coordination entre les enseignants au sein de leur propre établissement ou entre les différents acteurs au niveau du réseau. À ce titre, Bardram (1998) présente l'architecture du travail collaboratif de la manière suivante : la coordination, la coopération et la co-construction (Fig. 2).

Figure 2 : Les dynamiques du travail coopératif 

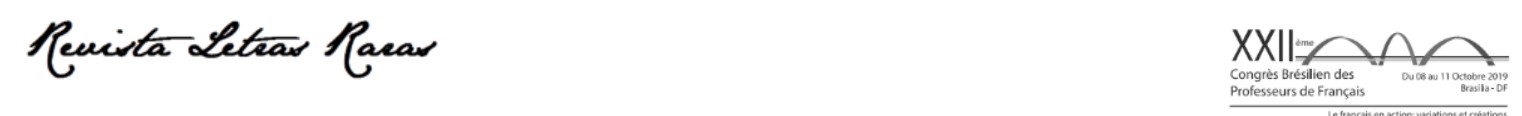

Actes du XXIlème Congrès brésilien des professeurs de français (dans Édition spéciale de la Revue Letras Raras 2020)

ISSN : 2317-2347 - v. 9, Dossier Spécial (2020)

Todo o conteúdo da RLR está licenciado sob Creative Commons Atribuição 4.0 Internacional

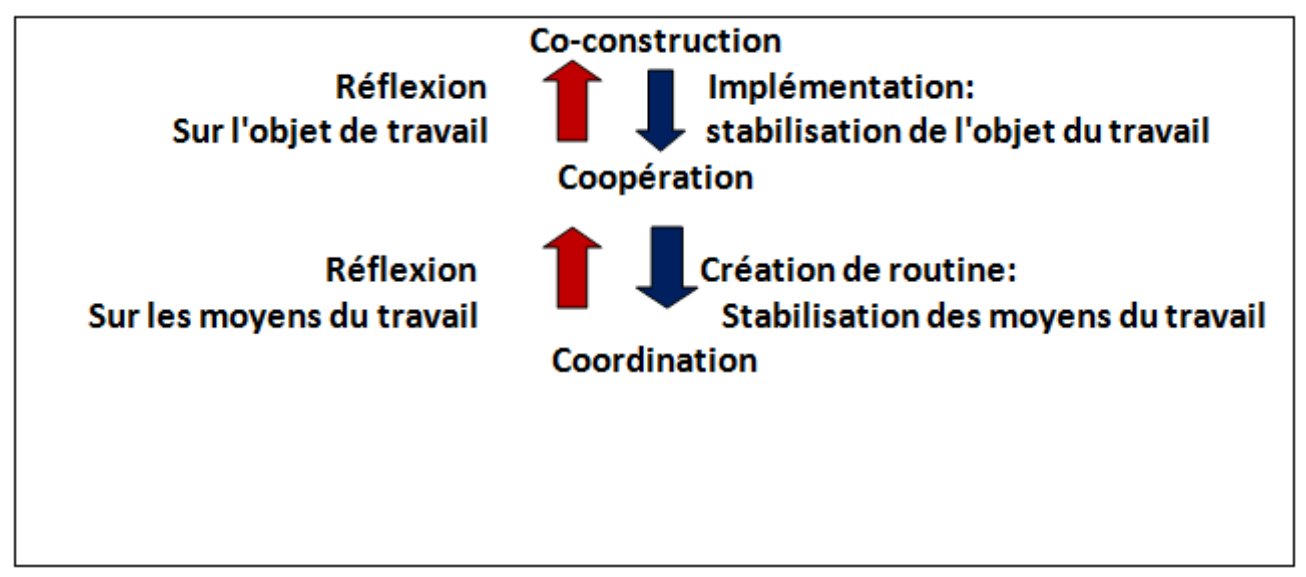

Source : BARDRAM, 1998, p. 11.

Selon la théorie présentée par Bardram (1998), les activités effectuées par les enseignants se distinguent selon leurs objets respectifs. De même, il souligne que les activités humaines sont ancrées dans un contexte socioculturel spécifique. II identifie trois structures hiérarchiques : la coordination, la coopération et la co-construction. La coordination correspond au niveau le plus bas du travail collaboratif dans la mesure où les acteurs sont des acteurs passifs qui exécutent un travail prescrit et souvent individuel.

Dans le deuxième niveau, celui de la coopération, leur travail consiste en un mode d'interaction centré sur l'objet. Les acteurs partagent ainsi l'objectif de l'activité collaborative. À la différence des acteurs dits " passifs", les acteurs du deuxième niveau sont actifs dans la mesure où ils font des ajustements selon l'objectif commun de l'activité.

Le troisième niveau, celui de la co-construction, correspond au plus haut niveau de l'activité coopérative : I'objet de l'activité est construit collectivement (BARDRAM, 1998, p. 11-12). II semblerait donc, que la coordination disciplinaire, le degré le plus faible de coopération, ne puisse pas vraiment garantir le développement professionnel de l'enseignant. Cette théorie est particulièrement importante car elle nous invite à nous interroger sur la nature des dynamiques de coopération et les niveaux de coopération qui favorisent le développement professionnel des enseignants.

En effet, la nécessité de la coopération entre les enseignants a été analysée par de nombreux 

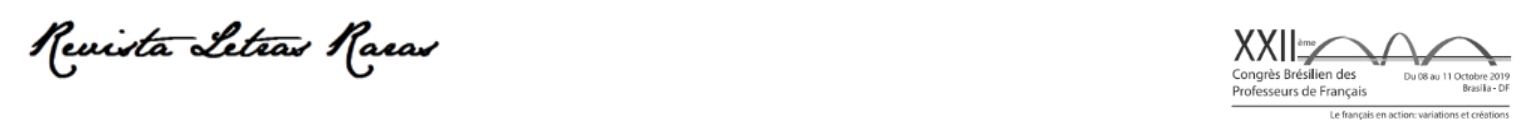

Actes du XXIlème Congrès brésilien des professeurs de français (dans Édition spéciale de la Revue Letras Raras 2020)

ISSN : 2317-2347 - v. 9, Dossier Spécial (2020)

Todo o conteúdo da RLR está licenciado sob Creative Commons Atribuição 4.0 Internacional

chercheurs en sciences de l'éducation (HUBERMAN, 1995 ; LIEBERMAN ; MILLER, 2001 ; FULLAN ; HARGREAVES, 2012) et également soulignée dans les travaux de Cochran-Smith et Lytle (1999; 2001). Ces derniers ont établi un cadre conceptuel qui s'articule autour de trois axes contribuant au développement professionnel de l'enseignant en cours d'emploi. Le cadre qu'ils appellent la source du savoir "Knowledge source ", permet d'appréhender la complexité de la construction du savoir enseignant. Selon Smith et Lytle (2001), la complexité du métier d'enseignant est liée à son obligation de s'engager dans plusieurs processus de construction de son propre savoir. Ces processus lui permettent de faire des changements efficaces dans ses pratiques. Le premier axe concerne le savoir pour la pratique "Knowledge for Practice ». Ce savoir se construit grâce aux stages de formation, à la lecture d'articles scientifiques, etc. En revanche, ces sources de savoir ne garantissent pas le transfert, ou l'intégration de ce nouveau savoir, dans les pratiques enseignantes.

Le deuxième axe concerne le savoir en pratique "Knowledge in practice ». Ce dernier se construit à travers l'expérimentation, ou l'application d'une nouvelle méthode, proposée lors d'une formation ou lors d'une conférence. Ce savoir est consolidé par la collaboration et le dialogue entre les enseignants. Cette dynamique d'interaction entre pairs peut générer une réflexion chez l'enseignant, améliorer la mise en œuvre d'une nouvelle méthode et favoriser la mutualisation d'un nouveau savoir.

Le troisième axe concerne le savoir de la pratique "Knowledge of practice ». II se focalise sur la capacité réflexive de l'enseignant qui remet en question ses méthodes d'enseignement et construit, grâce à la collaboration avec ses pairs, l'usage de plusieurs ressources, le questionnement sur l'impact de ses méthodes sur les apprentissages des élèves et son savoir de la pratique. La combinaison de ces trois axes constitue, selon Cochran-Smith et Lytle (2001), les trois sources du savoir qui conduisent à un développement professionnel efficace. Le développement professionnel dépend, entre autres, de l'articulation de ces trois axes.

Par ailleurs, Deutsch cité par Johnson et al. (2014) pense que la coopération entre les enseignants suppose l'existence d'une interdépendance entre les individus. En revanche, la nature de cette interdépendance peut constituer un levier ou, au contraire, un obstacle. II semble que l'existence de cette " interdépendance » entre les individus pourrait favoriser ou, au contraire, entraver le développement professionnel des enseignants. Deutsch propose trois types d'interdépendance. 

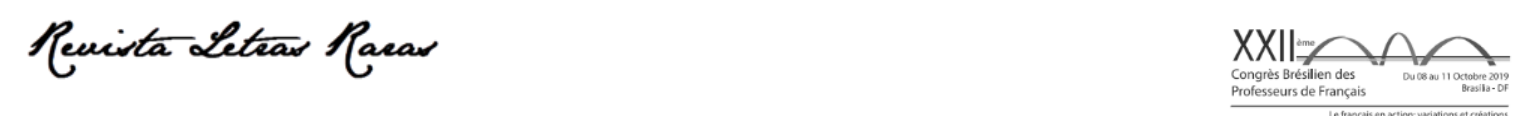

Actes du XXIlème Congrès brésilien des professeurs de français (dans Édition spéciale de la Revue Letras Raras 2020) ISSN : 2317-2347 - v. 9, Dossier Spécial (2020)

Todo o conteúdo da RLR está licenciado sob Creative Commons Atribuição 4.0 Internacional

Le premier type correspond à l'« interdépendance positive ». C'est une forme de coopération qui existe quand les objectifs à atteindre par tous les individus sont positivement corrélés. Par conséquent, les acteurs considèrent que l'atteinte de leurs objectifs dépend de celle des autres groupes. Le deuxième type d'interdépendance est celui de l'« interdépendance négative » qui désigne la compétition. Celle-ci existe quand les objectifs à atteindre par les individus sont négativement corrélés : chaque individu considère que sa réussite signifie l'échec des autres rivaux dans l'atteinte de leurs objectifs. L'interaction devient oppositionnelle 5 : « oppositional interaction » (Deutsch cité par Johnson, 2014, p. 4). Le troisième type d'interdépendance correspond à l'absence d'interdépendance : quand la "structure est individualiste " (Deutsch cité par Johnson 2014, p. 4), il n'y a pas de corrélation quant aux objectifs à atteindre par les individus. Chacun pense qu'il peut atteindre son objectif seul.

\section{Conclusion}

Au vu de ces résultats, l'enjeu majeur concernerait, d'une part, le réexamen des contenus du travail d'équipe et, d'autre part, la recherche de moyens efficaces pour que le réseautage dépasse le cercle restreint de l'équipe disciplinaire de l'établissement. Nous pouvons donc comprendre la nature de coopération qui pourrait exister entre les enseignants et entre les établissements scolaires. Une coopération qui favorise l'instauration d'une culture d'interdépendance.

C'est précisément cette culture de coopération et d'interdépendance qui marque l'histoire d'un groupe de professionnels qui devient une association, une fédération. Ce sont ces moments de rencontres scientifiques et d'échanges qui favorisent l'émergence des réseaux.

\section{Références}

\footnotetext{
${ }^{5}$ Traduction proposée par l'auteure : « Oppositional interaction » : interaction oppositionnelle
} 

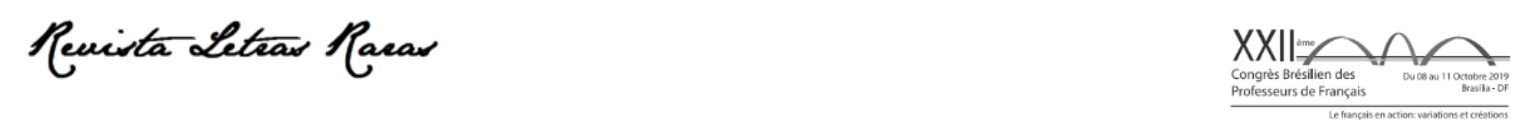

Actes du XXIlème Congrès brésilien des professeurs de français (dans Édition spéciale de la Revue Letras Raras 2020)

ISSN : 2317-2347 - v. 9, Dossier Spécial (2020)

Todo o conteúdo da RLR está licenciado sob Creative Commons Atribuição 4.0 Internacional

BARDRAM, J. Designing for the dynamics of cooperative work activities. Dans Proceedings of the ACM CSCW '98 conference on computer supported cooperative work. Association for comuting machiner, New York, p. 89-98, 1998.

BAUDRIT, A. L'apprentissage Coopératif Origines Et évolutions D' Une Méthode Pédagogique. Pédagogies En Développement. Bruxelles : De Boeck, 2005.

TARDIF Maurice, MARCEL Jean-François, DUPRIEZ Vincent \& PERISSET-BAGNOUD Danièle (dir.). Coordonner, Collaborer, Coopérer. De Nouvelles Pratiques Enseignantes." Revue Française De Pédagogie v. 165, n. 4, p. 149-51, 2008.

COCHRAN-SMITH, M. ; LYTLE, S. L. Beyond certainty: taking an inquiry stance on practice. Dans A. Lieberman et L. Miller (dir.), Teachers caught in the action: professional development that matters. New York: Teachers College Press, p. 45-58, 2001.

COCHRAN-SMITH, M. LYTLE, S. "Relationships of Knowledge and Practice: Teacher Learning in Communities." Review of Research in Education, v. 24 (1999), p. 249-305. Disponivel em: [http://www.jstor.org/stable/1167272]. Acesso em: 27 nov.2020.

DAY, C. Developing Teachers. London: Routledge, 1999.

DIONNE, L. La collaboration entre collègues comme mode de développement professionnel chez l'enseignant: une étude de cas. Thèse de doctorat inédite, Université du Québec à Montréal, Montréal, Québec, 2003.

FULLAN, M. "Leadership for the 21st Century: Breaking the Bonds of Dependency." Educational Leadership , v. 55, n. 7, p. 6-10, 1998.

GRANGEAT, M. Coopérations Et Partenariats Pour Enseigner: Pratiques De L'éducation Prioritaire. SCEREN, 2008.

HARGREAVES, A. ; FULLAN, M. Professional capital: transforming teaching in every school. New York : Teachers College, 2012.

HARGREAVES, A. ; FULLAN, M. What's worth fighting for out there? Toronto: Ontario Public School Teachers' Federation, New York: Teacher's College Press and Buckingham : Open University Press, 1998.

HUBERMAN, M. Networks that alter teaching: Conceptualizations, exchanges, and experiments. Teachers and Teaching, Theory and Practice, 1, p. 193-211, 1995.

JOHNSON, D. W., JOHNSON, R. T., \& Smith, K. A. Co- operative learning: Improving university instruction by basing practice on validated theory. Journal on Excellence in College Teaching, v. 25, 

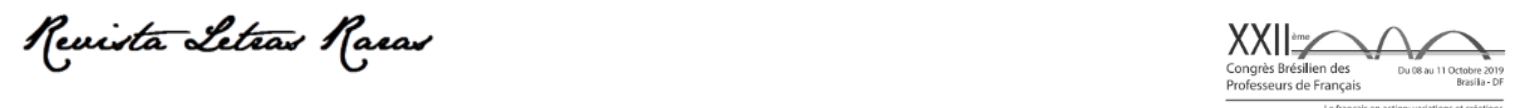

Actes du XXIlème Congrès brésilien des professeurs de français (dans Édition spéciale de la Revue Letras Raras 2020)

ISSN : 2317-2347 - v. 9, Dossier Spécial (2020)

Todo o conteúdo da RLR está licenciado sob Creative Commons Atribuição 4.0 Internacional

(3-4), p. 85-118, 2014.

LAFORTUNE, L.; LEPAGE, C. Guide pour l'accompagnement professionnel d'un changement. Québec : Presses universitaires du Québec, 2008.

LECLERC, M. Communauté d'apprentissage professionnelle : guide à l'intention des leaders scolaires. Appartient à la collection : Collection Éducation intervention, 35, Québec: Presses de I'Université du Québec, 2012.

LITTLE, J. W. The persistence of privacy: autonomy and initiative in teachers' professional relations. Teachers College Record, 91 (4), p. 509-36, 1990.

MARCEL, J-F, et al. Coordonner, collaborer, coopérer. De nouvelles pratiques enseignantes. De Boeck Supérieur, 2007

MEN. (2008). Ministère de l'Éducation nationale. Bulletin officiel. Arrêté relatif au référentiel de compétences des enseignants. Récupéré le 2 mars 2020 de [www.education.gouv.fr/]

PRIEUR, M. ; SANCHEZ, E. ; ALDON, G. Enseignement scientifique co-disciplinaire en classe de seconde : éléments à prendre en compte pour sa mise en œuvre. In M. GRANGEAT (Ed.), Les démarches d'investigation dans l'enseignement scientifique - Pratiques de classe, travail collectif enseignant, acquisitions des élèves. Lyon : Ecole Normale Supérieure de Lyon, p. 107-121, 2011.

SERIEYX, H., AZOULAY, H., Groupe CFC. Mettez du réseau dans vos pyramides : penser, organiser, vivre la structure en réseau. Paris : Village Mondial, 1996.

TARDIF, M. et LESSARD, C. Le Travail Enseignant Au Quotidien. Expérience, Interactions Humaines Et Dilemmes Professionnels. Bruxelles : De Boeck Université." Revue Des Sciences De L'éducation v. 27, n. 2, p. 448-49, 1999.

WILDAVSKY, A. On collaboration. Political Science and Politics, v. 19, p. 237-248, 1986. 